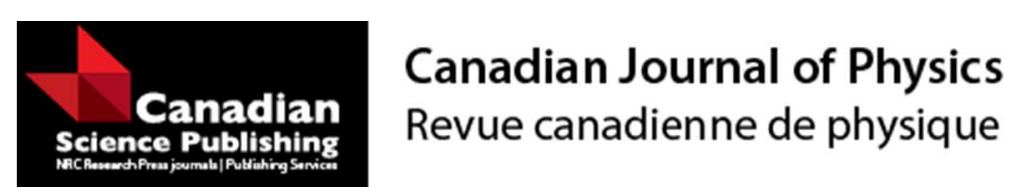

\title{
The upper critical magnetic field of holographic superconductor with conformally invariant power-Maxwell electrodynamics
}

\begin{tabular}{|r|l|}
\hline Journal: & Canadian Journal of Physics \\
\hline Manuscript ID & cjp-2016-0913 \\
\hline Manuscript Type: & Article \\
\hline Complete List of Authors: & $\begin{array}{l}\text { Sheykhi, Ahmad; Shiraz University } \\
\text { Shamsi, Fereshteh; Shiraz University } \\
\text { Davatolhagh, Saeed; Shiraz University }\end{array}$ \\
\hline Keyword: & $\begin{array}{l}\text { holographic superconductor, magnetic field, critical temperature, conformal } \\
\text { invariance, power-Maxwell }\end{array}$ \\
\hline \multicolumn{2}{|c}{} \\
\hline
\end{tabular}




\title{
The upper critical magnetic field of holographic superconductor with conformally invariant power-Maxwell electrodynamics
}

\author{
A. Sheykhi ${ }^{1,2 *}$, F. Shamsi ${ }^{1}$, and S. Davatolhagh ${ }^{1 \dagger}$ \\ 1 Physics Department and Biruni Observatory, College of Sciences, Shiraz University, Shiraz 71946, Iran \\ ${ }^{2}$ Research Institute for Astronomy and Astrophysics of Maragha (RIAAM), P.O. Box 55134-441, Maragha, Iran
}

\begin{abstract}
The properties of $(d-1)$-dimensional $s$-wave holographic superconductor in the presence of power-Maxwell field is explored. We study the probe limit in which the scalar and gauge fields do not backreact on the background geometry. Our study is based on the matching of solutions on the boundary and on the horizon at some intermediate point. At first, the case without external magnetic field is considered, and the critical temperature is obtained in terms of the charge density, the dimensionality, and the power-Maxwell exponent. Then, a magnetic field is turned on in the $d$-dimensional bulk which can influence the $(d-1)$-dimensional holographic superconductor at the boundary. The phase behavior of the corresponding holographic superconductor is obtained by computing the upper critical magnetic field in the presence of power-Maxwell electrodynamics, characterized by the power exponent $q$. Interestingly, it is observed that in the presence of magnetic field, the physically acceptable phase behavior of the holographic superconductor is obtained for $q=d / 4$, which guaranties the conformal invariance of the power-Maxwell Lagrangian. The case of physical interest in five spacetime dimensions $(d=5$, and $q=5 / 4)$ is considered in detail, and compared with the results obtained for the usual Maxwell electrodynamics $q=1$ in the same dimensions.
\end{abstract}

PACS numbers: 11.25.Tq, 04.70.Bw, 74.20.-z

Keywords: holographic superconductor; magnetic field; critical temperature

\section{INTRODUCTION}

The correspondence between the gravity in a $d$-dimensional anti-de Sitter (AdS) spacetime and the conformal field theory (CFT) residing on the $(d-1)$-dimensional boundary of this spacetime, provides a powerful tool for studying strongly coupled systems [1]. The gauge/gravity duality, which relates strongly interacting gauge theories to theories of gravity in higher dimensions, has opened a new window to study many different strongly interacting condensed matter systems [2]. In Ref. [3], a model for dual description of a holographic superconductor was proposed. The model was shown to exhibit a critical point $T_{c}$ at which the system goes into a superconducting phase. The properties of this phase have been thoroughly studied $[2,3]$, showing a strong resemblance with those of a Type II superconductor. Till date, a number of attempts have been made, mostly numerical, in order to understand various properties of holographic superconductors in the framework of the usual Maxwell electromagnetic theory [3-16].

It is important to investigate the issue of response to an external magnetic field in the context of holographic superconductors [17], which also is of central significance in the general field of superconductivity. It is observed that when immersed in an external magnetic field, ordinary superconductors expel the magnetic flux lines thereby exhibiting perfect diamagnetism when the temperature is lowered through $T_{c}$, which is called the Meissner effect [18]. In fact depending on their behavior in the presence of an external magnetic field, the superconductors are classified into two general categories, namely type I and type II superconductors. In type I superconductors, when the external magnetic field $B$ reaches a critical value $B_{c}$ there occurs an abrupt (first order) phase transition from the superconducting phase to the normal phase. On the other hand, in type II superconductors that are directly relevant to our discussion, there happens to be a continuous (second order) phase transition and the material ceases to superconduct for $B>B_{\mathrm{c} 2}$, where $B_{\mathrm{c} 2}$ is called the upper critical field of type II superconductor. In the case of the continuous phase transition in type II superconductor, the order parameter is small near $B_{\mathrm{c} 2}$ and vanishes continuously as $B \rightarrow B_{\mathrm{c} 2}$.

For the holographic superconductor in the probe limit, we neglect the backreaction of the scalar field on the background geometry. As a result, the holographic superconductor is not able to repel the background magnetic field, which holds a strong resemblance with type II superconductor in its mixed phase. Instead the scalar condensate adjusts itself such that it only fills a finite strip in the plane, thus reducing the total magnetic field passing through it. In other words, the effect of the external magnetic field is such that it always tries to reduce the condensate. The

\footnotetext{
* asheykhi@shirazu.ac.ir

$\dagger$ davatolhagh@susc.ac.ir
} 
numerical studies indicate that the superconducting phase disappears above an upper critical value of the applied magnetic field $B>B_{\mathrm{c} 2}[17,19]$.

It is well known that the properties of holographic superconductor depend on the behavior of the electromagnetic field coupled with the charged scalar field. The effects of nonlinear electrodynamics on the critical temperature and condensation parameters of $s$-wave holographic superconductors have been investigated in [20-22]. Motivated by the recent studies [23-29], and the fact that within the framework of AdS/CFT correspondence a different electromagnetic action is expected to modify the dynamics of the dual theory, in this paper we investigate the behavior of holographic superconductor with power-Maxwell field in the background of a $d$-dimensional Schwarzschild AdS black hole, bearing in mind that in $d$-dimensional spacetime the energy-momentum tensor of conformally invariant Maxwell field is traceless provided we take $q=d / 4$ where $q$ is the power parameter of the power-Maxwell electrodynamics [30, 31]. In the absence of external magnetic field, and in the background of $d$-dimensional AdS black hole, the properties of $s$-wave holographic superconductors coupled to power-Maxwell electrodynamics have been explored in [32-34]. Other studies on the holographic superconductors have been carried out in [35].

The rest of this paper is organized as follows. In Sec. II, we present the holographic dual of $d$-dimensional Schwarzschild AdS black hole by introducing a complex charged scalar field coupled with the power Maxwell field and explore the relation between critical temperature and charge density. In Sec. III, the magnetic field effect on holographic superconductor with power Maxwell electrodynamics is considered. The paper is concluded with a discussion and summary in section IV.

\section{HOLOGRAPHIC SUPERCONDUCTOR WITH POWER MAXWELL FIELD}

We consider the $d$-dimensional action of Einstein gravity in the presence of power-Maxwell field and a charged complex scalar field which is given by

$$
S=\int d^{d} x \sqrt{-g}\left[R-2 \Lambda-\beta\left(F_{\mu \nu} F^{\mu \nu}\right)^{q}-\left|\nabla_{\mu} \psi-i q A_{\mu} \psi\right|^{2}-m^{2}|\psi|^{2}\right],
$$

where $R$ is the Ricci scalar, $F_{\mu \nu}$ is the electromagnetic field tensor, $A_{\mu}$ and $\psi$ are the gauge and scalar field, $\beta$ is the coupling constant, $q$ is the power parameter of the power-Maxwell field, and the cosmological constant is

$$
\Lambda=-\frac{(d-1)(d-2)}{2 l^{2}},
$$

where $l$ is the AdS radius of spacetime. For $\beta=1 / 4$ and $q=1$ the power-Maxwell Lagrangian $\mathcal{L}=-\beta\left(F_{\mu \nu} F^{\mu \nu}\right)^{q}$ reduces to the usual Maxwell Lagrangian. Besides, for $q=d / 4$, the energy-momentum tensor of the power-Maxwell Lagrangian is traceless in all dimensions [30]. It is also easy to check that, under a conformal transformation which acts on the fields as $g_{\mu \nu} \rightarrow \Omega^{2} g_{\mu \nu}$ and $A_{\mu} \rightarrow A_{\mu}$, the power-Maxwell Lagrangian $\mathcal{L}=-\beta\left(F_{\mu \nu} F^{\mu \nu}\right)^{q}$ remains unchanged provided $q=d / 4$ [30]. The metric of a planar Schwarzschild AdS black hole in $d$-dimensional spacetime is

$$
d s^{2}=-f(r) d t^{2}+\frac{d r^{2}}{f(r)}+r^{2} d x_{i} d x^{i}
$$

where

$$
f(r)=r^{2}\left(1-\frac{r_{+}^{d-1}}{r^{d-1}}\right)
$$

$r_{+}$is the horizon radius, and we have taken the AdS radius equal to one, i.e. $l=1$. The Hawking temperature is given by

$$
T=\frac{f^{\prime}\left(r_{+}\right)}{4 \pi}=\frac{(d-1) r_{+}}{4 \pi} .
$$

We consider the following ansatz for the gauge and the scalar fields, respectively [3]

$$
A_{\mu}=\phi(r) d t, \quad \psi=\psi(r) .
$$

From the action in Eq. (1), the equations of motion are given by

$$
\phi^{\prime \prime}+\left(\frac{d-2}{2 q-1}\right) \frac{\phi^{\prime}}{r}+\frac{\phi \psi^{2} \phi^{2-2 q}}{(-2)^{q} \beta q(2 q-1) f}=0
$$




$$
\psi^{\prime \prime}+\left(\frac{f^{\prime}}{f}+\frac{d-2}{r}\right) \psi^{\prime}+\left(\frac{\phi^{2}}{f^{2}}-\frac{m^{2}}{f}\right) \psi=0,
$$

where the prime denotes derivative with respect to $r$. At the event horizon of the black hole $r=r_{+}$, the regularity gives the boundary conditions for $\psi(r)$ and $\phi(r)$ as [3]

$$
\phi\left(r_{+}\right)=0, \quad \psi\left(r_{+}\right)=\frac{(d-1) r_{+}}{m^{2}} \psi^{\prime}\left(r_{+}\right) .
$$

At the asymptotic AdS boundary $(r \rightarrow \infty)$, the solutions of Eqs. (7) and (8) behave like

$$
\begin{aligned}
\psi & \approx \frac{\psi_{-}}{r^{\lambda_{-}}}+\frac{\psi_{+}}{r^{\lambda_{+}}}, \\
\phi & \approx \mu-\frac{\rho^{\frac{1}{2 q-1}}}{r^{\frac{d-2}{2 q-1}-1}},
\end{aligned}
$$

with

$$
\lambda_{ \pm}=\frac{1}{2}\left[(d-1) \pm \sqrt{(d-1)^{2}+4 m^{2}}\right],
$$

where $\mu$ and $\rho$ are interpreted as the chemical potential and charge density in the dual field theory, respectively [3] By changing the variable $z=r_{+} / r$, the equations of motion (7) and (8) become

$$
\begin{gathered}
\phi^{\prime \prime}+\left(\frac{4 q-d}{2 q-1}\right) \frac{\phi^{\prime}}{z}+\frac{\phi \psi^{2} \phi^{2-2 q} r_{+}^{2 q}}{2^{q}(-1)^{3 q} \beta q(2 q-1) f z^{4 q}}=0, \\
\psi^{\prime \prime}+\left(\frac{f^{\prime}}{f}-\frac{d-4}{z}\right) \psi^{\prime}+\frac{r_{+}^{2}}{z^{4}}\left(\frac{\phi^{2}}{f^{2}}-\frac{m^{2}}{f}\right) \psi=0,
\end{gathered}
$$

where the prime now indicates the derivative with respect to the new coordinate $z$. The asymptotic boundary conditions for the scalar field $\psi(z)$ and the scalar potential $\phi(z)$ now become

$$
\begin{gathered}
\phi=\mu-\frac{\rho^{\frac{1}{2 q-1}}}{r_{+}^{\frac{d-2}{2 q-1}-1}} z^{\frac{d-2}{2 q-1}-1}, \\
\psi=J_{-} z^{\lambda_{-}}+J_{+} z^{\lambda_{+}} .
\end{gathered}
$$

Following [3, 6], we can impose the boundary condition that either $J_{+}$or $J_{-}$vanishes. Hereafter, we consider the case with $J_{+}=0$. The case with $J_{-}=0$ was already studied in [29], although their final expression for critical magnetic field seems to be in error.

Now, anticipating the matching technique [5, 7, 10, 36-38], first we consider the solutions of the gauge field $\phi(z)$ and the scalar field $\psi(z)$, using the boundary conditions from Eqs. (9) and (10) near the horizon $(z=1)$. Both $\psi(z)$ and $\phi(z)$ are Taylor expanded near the horizon $(z=1)$ as

$$
\begin{gathered}
\phi(z)=\phi(1)-\phi^{\prime}(1)(1-z)+\frac{1}{2} \phi^{\prime \prime}(1)(1-z)^{2}+\ldots \\
\approx-\phi^{\prime}(1)(1-z)+\frac{1}{2} \phi^{\prime \prime}(1)(1-z)^{2} \\
\psi(z)=\psi(1)-\psi^{\prime}(1)(1-z)+\frac{1}{2} \psi^{\prime \prime}(1)(1-z)^{2}+\ldots
\end{gathered}
$$

where without loss of generality we choose $\phi^{\prime}(1)<0$ and $\psi(1)>0$. On the other hand, near horizon $(z=1)$ from Eqs. (13) and (14), and using $f^{\prime}(1)=-(d-1) r_{+}^{2}$ and $f^{\prime \prime}(1)=6 r_{+}^{2}-(d-3)(d-4) r_{+}^{2}$, we obtain

$$
\phi^{\prime \prime}(1)=\left(\frac{d-4 q}{2 q-1}\right) \phi^{\prime}(1)+\frac{\psi^{2}(1) r_{+}^{2 q-2}}{(-1)^{3 q} 2^{q} \beta q(2 q-1)(d-1)}\left[\phi^{\prime}(1)\right]^{3-2 q},
$$




$$
\psi^{\prime \prime}(1)=\frac{m^{2}}{d-1}\left(1+\frac{m^{2}}{2(d-1)}\right) \psi(1)-\frac{\phi^{2}(1) \psi(1)}{2 r_{+}^{2}(d-1)^{2}} .
$$

Substituting Eqs. (19) and (20) in Eqs. (17) and (18), we finally obtain

$$
\begin{gathered}
\phi(z) \approx-\phi^{\prime}(1)(1-z)+\frac{1}{2}\left\{\frac{d-4 q}{2 q-1}+\frac{\psi^{2}(1)\left[\phi^{\prime}(1)\right]^{2-2 q} r_{+}^{2 q-2}}{(-1)^{3 q} 2^{q} \beta q(2 q-1)(d-1)}\right\} \phi^{\prime}(1)(1-z)^{2}, \\
\psi(z) \approx\left(1+\frac{m^{2}}{d-1}\right) \psi(1)-\frac{m^{2}}{d-1} z \psi(1)+\frac{1}{2}\left\{\frac{m^{2}}{d-1}\left(1+\frac{m^{2}}{2(d-1)}\right)-\frac{\phi^{\prime 2}(1)}{2 r_{+}^{2}(d-1)^{2}}\right\} \psi(1)(1-z)^{2} .
\end{gathered}
$$

Now by matching these two sets of asymptotic solutions at some intermediate point $z=z_{m}$, namely matching Eqs. (15) and (16), respectively, with Eqs. (21) and (22), we arrive at the following set of equations,

$$
\begin{gathered}
\mu-\frac{\rho^{\frac{1}{2 q-1}}}{r_{+}^{\frac{d-2}{2 q-1}-1}} z_{m}^{\frac{d-2}{2 q-1}-1}=\omega\left(1-z_{m}\right)-\frac{1}{2}\left\{\frac{d-4 q}{2 q-1}+\frac{\alpha^{2}(-\omega)^{2-2 q} r_{+}^{2 q-2}}{(-1)^{3 q} 2^{q} \beta q(2 q-1)(d-1)}\right\} \omega\left(1-z_{m}\right)^{2}, \\
J_{-} z_{m}^{\lambda_{-}}=\left(1+\frac{m^{2}}{d-1}\right) \alpha-\frac{m^{2}}{d-1} z_{m} \alpha+\frac{1}{2}\left\{\frac{m^{2}}{d-1}\left(1+\frac{m^{2}}{2(d-1)}\right)-\frac{\omega^{2}}{2 r_{+}^{2}(d-1)^{2}}\right\} \alpha\left(1-z_{m}\right)^{2},
\end{gathered}
$$

where we have defined $\alpha \equiv \psi(1)$ and $\omega \equiv-\phi^{\prime}(1)(\alpha, \omega>0)$. Note that according to the matching method, not only functions $\phi(z)$ and $\psi(z)$ should match at $z_{m}$, but their derivatives must also match at intermediate point $z_{m}$. The latter implies that we have the following two equations

$$
\begin{gathered}
\left(\frac{d-2}{2 q-1}-1\right) \frac{\rho^{\frac{1}{2 q-1}}}{r_{+}^{\frac{d-2}{2 q-1}-1}} z_{m}^{\frac{d-2}{2 q-1}-2}=\omega-\left\{\frac{d-4 q}{2 q-1}+\frac{\alpha^{2}(-\omega)^{2-2 q} r_{+}^{2 q-2}}{(-1)^{3 q} 2^{q} \beta q(2 q-1)(d-1)}\right\} \omega\left(1-z_{m}\right), \\
\lambda_{-} J_{-} z_{m}^{\lambda_{-}-1}=-\frac{m^{2}}{d-1} \alpha-\left\{\frac{m^{2}}{d-1}\left(1+\frac{m^{2}}{2(d-1)}\right)-\frac{\omega^{2}}{2 r_{+}^{2}(d-1)^{2}}\right\} \alpha\left(1-z_{m}\right) .
\end{gathered}
$$

From Eq. (25), after using Eq. (5), we find

$$
\alpha^{2}=\frac{(-1)^{5 q-1} 2^{q} q \beta(d-1)}{\left(1-z_{m}\right) \tilde{\omega}^{2(1-q)}}\left[\left(z_{m}-1\right) d-1+\left(6-4 z_{m}\right) q\right]\left(\frac{T_{c}}{T}\right)^{\left(\frac{d-2}{2 q-1}\right)}\left[1-\left(\frac{T}{T_{c}}\right)^{\left(\frac{d-2}{2 q-1}\right)}\right]
$$

where $\tilde{\omega}=\omega / r_{+}$, and $T_{c}$ is obtained as

$$
T_{c}=\kappa \rho^{\frac{1}{d-2}}
$$

where

$$
\kappa=\frac{(d-1) z_{m}^{\frac{d-4 q}{d-2}}}{4 \pi \tilde{\omega}^{\frac{2 q-1}{d-2}}}\left[\frac{(d-2 q-1)}{\left(z_{m}-1\right) d-1+\left(6-4 z_{m}\right) q}\right]^{\frac{2 q-1}{d-2}} .
$$

From Eqs. (24) and (26), we have

$$
\begin{gathered}
\tilde{\omega}=\sqrt{m^{4}+\frac{2(d-1)}{\left(1-z_{m}\right)\left(2 z_{m}+\lambda_{-}\left(1-z_{m}\right)\right)}\left[m^{2}\left(\lambda_{-}\left(z_{m}^{2}-4 z_{m}+3\right)-2 z_{m}\left(z_{m}-2\right)\right)+2 \lambda_{-}(d-1)\right]}, \\
J_{-}=\frac{2(d-1)+m^{2}\left(1-z_{m}\right)}{z_{m}^{\left(\lambda_{-}-1\right)}(d-1)\left(2 z_{m}+\lambda_{-}\left(1-z_{m}\right)\right)} \alpha .
\end{gathered}
$$


Near the critical point $T \sim T_{c}$, after using Eq. (27) and the definition of distance parameter $t \equiv 1-T / T_{c}$, we get

$$
\alpha=\sqrt{\mathcal{A}} \sqrt{\frac{d-2}{2 q-1} t}
$$

where

$$
\mathcal{A}=\frac{(-1)^{5 q-1} 2^{q} q \beta(d-1)}{\left(1-z_{m}\right) \tilde{\omega}^{2(1-q)}}\left[\left(z_{m}-1\right) d-1+\left(6-4 z_{m}\right) q\right] .
$$

Finally, combining Eqs. (5), (31), and (32) near the critical temperature, the condensation operator can be calculated as

$$
<\mathcal{O}_{-}>=\sqrt{2} r_{+} J_{-}=\gamma T_{c} \sqrt{1-\frac{T}{T_{c}}}
$$

where

$$
\begin{gathered}
\gamma=\frac{4 \sqrt{2} \pi \sqrt{\mathcal{A}}}{(d-1)^{2}} \sqrt{\frac{d-2}{2 q-1}} \frac{2(d-1)+m^{2}\left(1-z_{m}\right)}{z_{m}^{\left(\lambda_{-}-1\right)}\left[2 z_{m}+\lambda_{-}\left(1-z_{m}\right)\right]} . \\
\qquad \begin{array}{|c|c|c|}
\hline d & q & \kappa \\
\hline 4 & 1 & 0.142 \\
\hline 5 & 5 / 4 & 0.197 \\
\hline 6 & 6 / 4 & 0.231 \\
\hline 7 & 7 / 4 & 0.259 \\
\hline 8 & 2 & 0.285 \\
\hline
\end{array}
\end{gathered}
$$

Table 1: The values of $\kappa=T_{c} / \rho^{1 /(d-2)}$ for different values of power parameter $q=d / 4$, with $z_{m}=0.5$ and $m^{2}=-2$.

We see from Table 1 that the critical temperature increases with increasing power parameter $q=d / 4$, or dimensions $d$, that is consistent with the general theory of critical phenomena. There are two cases of physical interest in Table 1. The case of $d=4$ corresponds to the (2+1)-dimensional holographic superconductor in the usual Maxwell field $q=1$. The other case of physical interest is $d=5$, which corresponds to a $(3+1)$-dimensional holographic superconductor with conformally invariant power-Maxwell field $q=5 / 4$.

\section{EFFECT OF EXTERNAL MAGNETIC FIELD}

According to the gauge/gravity duality, the asymptotic value of magnetic field in the bulk, corresponds to the magnetic field in the boundary field theory: $B(x)=F_{x y}(x, z \rightarrow 0)[17,23]$. Considering the fact that near the upper critical magnetic field of the continuous phase transition $B_{\mathrm{c} 2}$, the condensate order parameter is small, we can therefore consider the scalar field $\psi$ as a small perturbation near $B_{\mathrm{c} 2}$. This allows us to adopt the following ansatz for the gauge field and the scalar field [17, 23, 24, 29]

$$
A_{t}=\phi(z), \quad A_{y}=B x, \quad \psi=\psi(x, z) .
$$

With this, the equation for the scalar field $\psi$ becomes

$$
\psi^{\prime \prime}(x, z)+\left(\frac{f^{\prime}(z)}{f(z)}-\frac{d-4}{z}\right) \psi^{\prime}(x, z)-\frac{m^{2} r_{+}^{2} \psi(x, z)}{z^{4} f(z)}+\frac{r_{+}^{2} \phi^{2}(z) \psi(x, z)}{z^{4} f^{2}(z)}+\frac{1}{z^{2} f(z)}\left(\partial_{x}^{2} \psi-B^{2} x^{2} \psi\right)=0 .
$$

In order to solve Eq. (37), we take the following separable form

$$
\psi(x, z)=X(x) R(z) .
$$

Substituting Eq. (38) into Eq. (37), we arrive at

$$
z^{2} f(z)\left[\frac{R^{\prime \prime}}{R}+\left(\frac{f^{\prime}(z)}{f(z)}-\frac{d-4}{z}\right) \frac{R^{\prime}}{R}+\frac{r_{+}^{2} \phi^{2}}{z^{4} f^{2}}-\frac{m^{2} r_{+}^{2}}{z^{4} f}\right]-\left[-\frac{X^{\prime \prime}}{X}+B^{2} x^{2}\right]=0 .
$$


$X(x)$ is governed by the equation of a harmonic oscillator with frequency determined by $B$ :

$$
-X^{\prime \prime}(x)+B^{2} x^{2} X(x)=\lambda_{n} B X(x),
$$

where $\lambda_{n}=2 n+1$. For $n=0, R(z)$ satisfies

$$
R^{\prime \prime}(z)+\left(\frac{f^{\prime}(z)}{f(z)}-\frac{d-4}{z}\right) R^{\prime}(z)-\frac{m^{2} r_{+}^{2} R(z)}{z^{4} f(z)}+\frac{r_{+}^{2} \phi^{2}(z) R(z)}{z^{4} f^{2}(z)}=\frac{B R(z)}{z^{2} f(z)} .
$$

Now at the horizon $z=1$, and using Eq. (41), $\phi(1)=0, f^{\prime}(1)=-(d-1) r_{+}^{2}$, and $f^{\prime \prime}(1)=6 r_{+}^{2}-(d-3)(d-4) r_{+}^{2}$, we obtain

$$
\begin{gathered}
R^{\prime}(1)=-\left(\frac{B}{(d-1) r_{+}^{2}}+\frac{m^{2}}{d-1}\right) R(1), \\
R^{\prime \prime}(1)=\left\{\frac{m^{2}}{d-1}\left(1+\frac{B}{(d-1) r_{+}^{2}}+\frac{m^{2}}{2(d-1)}\right)+\frac{B^{2}}{2(d-1)^{2} r_{+}^{4}}-\frac{\phi^{\prime 2}(1)}{2(d-1)^{2} r_{+}^{2}}\right\} R(1) .
\end{gathered}
$$

In the asymptotic region $(z \rightarrow 0)$, the solution behaves as

$$
R(z)=J_{-} z^{\lambda_{-}}+J_{+} z^{\lambda_{+}} .
$$

In the presence of external magnetic field, we use the matching method and the Taylor expansion of $R(z)$ near the horizon

$$
R(z)=R(1)-R^{\prime}(1)(1-z)+\frac{1}{2} R^{\prime \prime}(1)(1-z)^{2}+\cdots .
$$

Substituting Eqs. (42) and (43) in Eq. (45), we have

$$
\begin{aligned}
R(z) \approx & R(1)+(1-z)\left(\frac{B}{(d-1) r_{+}^{2}}+\frac{m^{2}}{d-1}\right) R(1) \\
& +\frac{1}{2}\left[\frac{m^{2}}{d-1}\left(1+\frac{B}{(d-1) r_{+}^{2}}+\frac{m^{2}}{2(d-1)}\right)+\frac{B^{2}}{2(d-1)^{2} r_{+}^{4}}-\frac{\phi^{2}(1)}{2(d-1)^{2} r_{+}^{2}}\right] R(1)(1-z)^{2} .
\end{aligned}
$$

The two solutions given in Eq. (44) and Eq. (46) are connected smoothly at an intermediate point $z_{m}$. Thus, we find that

$$
\begin{gathered}
J_{-} z_{m}^{\lambda_{-}}=R(1)+\left(1-z_{m}\right)\left(\frac{B}{(d-1) r_{+}^{2}}+\frac{m^{2}}{d-1}\right) R(1)+\frac{1}{2}\left\{\frac{m^{2}}{d-1}\left(1+\frac{B}{(d-1) r_{+}^{2}}+\frac{m^{2}}{2(d-1)}\right)\right. \\
\left.+\frac{B^{2}}{2(d-1)^{2} r_{+}^{4}}-\frac{\phi^{\prime 2}(1)}{2(d-1)^{2} r_{+}^{2}}\right\} R(1)\left(1-z_{m}\right)^{2} \\
\lambda_{-} J_{-} z_{m}^{\lambda_{-}-1}=-\left(\frac{B}{(d-1) r_{+}^{2}}+\frac{m^{2}}{d-1}\right) R(1)-\left\{\frac{m^{2}}{d-1}\left(1+\frac{B}{(d-1) r_{+}^{2}}+\frac{m^{2}}{2(d-1)}\right)\right. \\
\left.+\frac{B^{2}}{2(d-1)^{2} r_{+}^{4}}-\frac{\phi^{\prime 2}(1)}{2(d-1)^{2} r_{+}^{2}}\right\} R(1)\left(1-z_{m}\right) .
\end{gathered}
$$

From the above equations, we get

$$
B^{2}+2 n r_{+}^{2} B+p r_{+}^{4}-\phi^{\prime 2}(1) r_{+}^{2}=0
$$

where

$$
n=m^{2}+2(d-1)\left\{\frac{\lambda_{-}\left(1-z_{m}\right)+z_{m}}{\left(1-z_{m}\right)\left[\left(2-\lambda_{-}\right) z_{m}+\lambda_{-}\right]}\right\},
$$




$$
p=m^{4}+\frac{2(d-1)\left[2 \lambda_{-}(d-1)+m^{2}\left(\left(3-4 z_{m}+z_{m}^{2}\right) \lambda_{-}-2 z_{m}\left(z_{m}-2\right)\right)\right]}{\left(1-z_{m}\right)\left[\left(2-\lambda_{-}\right) z_{m}+\lambda_{-}\right]} .
$$

It is easy to see that Eq. (49) has a solution

$$
B=\sqrt{\phi^{\prime 2}(1) r_{+}^{2}+r_{+}^{4}\left(n^{2}-p\right)}-n r_{+}^{2} .
$$

Now consider the case for which the value of the external magnetic field is very close to the upper critical value, i.e. $B \sim B_{\mathrm{c} 2}$. This implies a vanishingly small condensate, and therefore we can ignore all the quadratic terms in $\psi$. With this approximation, from Eq. (13) we obtain

$$
\phi^{\prime \prime}(z)-\frac{1}{z}\left(\frac{d-2}{2 q-1}-2\right) \phi^{\prime}(z)=0
$$

From the above equation and using Eq. (15), we arrive at

$$
\phi^{\prime 2}(1) r_{+}^{2}=\frac{\rho^{\frac{2}{2 q-1}}}{r_{+}^{\frac{2(d-2)}{2 q-1}}} r_{+}^{4}\left(\frac{d-2}{2 q-1}-1\right)^{2}
$$

From Eqs. (28) and (29), we have

$$
\rho=\frac{\left[4 \pi T_{c}(0)\right]^{(d-2)} \tilde{\omega}^{2 q-1}}{z_{m}^{(d-4 q)}(d-1)^{(d-2)}\left[\frac{d-2 q-1}{\left(z_{m}-1\right) d-1+\left(6-4 z_{m}\right) q}\right]^{2 q-1}} .
$$

On substituting Eqs. (54) and (55) into Eq. (52), we finally obtain

$$
\begin{aligned}
\frac{B_{\mathrm{c} 2}(T)}{T_{c}^{2}(0)}= & \frac{16 \pi^{2}}{(d-1)^{2}}\left(\frac{T}{T_{c}(0)}\right)^{\frac{4 q-d}{2 q-1}}\left\{\sqrt{\frac{\tilde{\omega}^{2}\left[\left(z_{m}-1\right) d-1+\left(6-4 z_{m}\right) q\right]^{2}}{(2 q-1)^{2} z_{m}^{\frac{2(d-4 q)}{2 q-1}}}+\left(n^{2}-p\right)\left(\frac{T}{T_{c}(0)}\right)^{\frac{2 d-4}{2 q-1}}}\right. \\
& \left.-n\left(\frac{T}{T_{c}(0)}\right)^{\frac{d-2}{2 q-1}}\right\} .
\end{aligned}
$$

This result makes clear the dependence of the upper critical field on the temperature and the power parameter of the power-Maxwell electrodynamics. The phase diagram given by Eq. (56) is plotted in Figs. 1 and 2 for different values of dimensionality $d$ (or power parameter $q=d / 4$ ) and different $m^{2}$, respectively. It is clear from Figs. 1 and 2 that, consistent with the phenomenology and the Ginzburg-Landau theory of superconductivity, $B_{\mathrm{c} 2}(T)$ vanishes linearly as $T \rightarrow T_{c}(0)$. Figure 3 shows the behavior of $B_{\mathrm{c} 2}$ as a function of $T$ for different values of $T_{c}(0)$, i.e. the transition temperature in absence of magnetic field. It is evident from Fig. 3 that $B_{\mathrm{c} 2}(0)$ increases with $T_{c}(0)$, which is again consistent with the phenomenology of superconductivity. It is worth noting that the reasonable behavior of $B_{\mathrm{c} 2}$ in Figs. 1-3, which is consistent with the phenomenology of superconductors, is observed provided we take $q=d / 4$. For example in $d=5$ dimensions that corresponds to a $(3+1)$-dimensional holographic superconductor, we should take $q=5 / 4$ in order to have reasonable $B_{\mathrm{c} 2}$ in the range of $T \leq T_{c}(0)$. This implies that among all power-Maxwell theories with various parameters $q$, those which are conformally invariant lead to the magnetic field behavior of physical interest for holographic superconductors. This may be understood as follows. For the conformally invariant power-Maxwell theory, not only the energy-momentum tensor is traceless, but also the electromagnetic fields in higher dimensions have the same behavior as in the (well established) case of $(2+1)$-dimensional holographic superconductor dual of 4-dimensional gravity [31]. For example, for the usual Maxwell field in $d$ dimensions, the electric field of charged point-like particle behaves as $E(r) \sim 1 / r^{d-2}$, while for the conformally invariant power-Maxwell field in $d$ dimensional spacetime, the electric field of point charge is independent of the dimensionality and varies as $E(r) \sim 1 / r^{2}$, exactly like in four dimensions [31]. In other words, for conformally invariant Maxwell field, the magnetic field in the $d$-dimensional bulk and on its $(d-1)$-dimensional boundary has the same behavior as in the usual holographic superconductor in $d=4$ spacetime.

In order to clarify the above argument, let us have a closer look at expression (56). For conformally invariant case $q=d / 4$, the critical magnetic field in $d$-dimensions reduces to

$$
\frac{B_{\mathrm{c} 2}(T)}{T_{c}^{2}(0)}=\frac{16 \pi^{2}}{(d-1)^{2}}\left[\sqrt{\tilde{\omega}^{2}+\left(n^{2}-p\right)\left(\frac{T}{T_{c}(0)}\right)^{4}}-n\left(\frac{T}{T_{c}(0)}\right)^{2}\right] .
$$




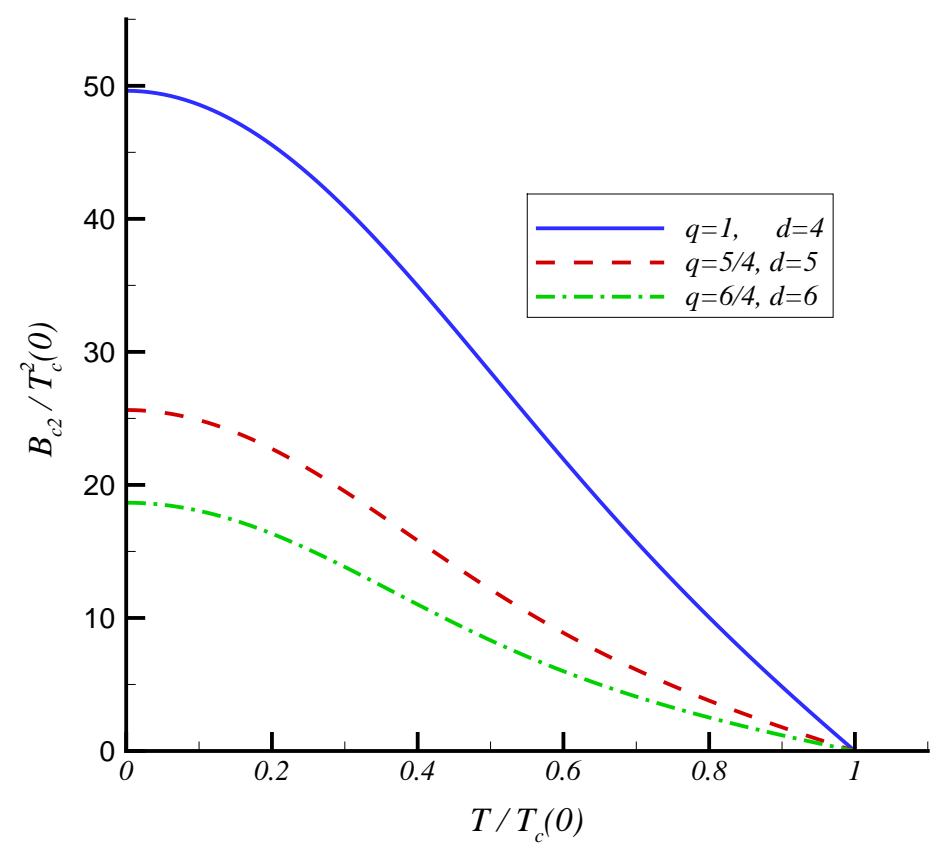

FIG. 1: The behavior of $B_{\mathrm{c} 2} / T_{c}^{2}(0)$ in terms of $T / T_{c}(0)$ for different values of $q$ and $d$, with $z_{m}=0.5$ and $m^{2}=-2$.

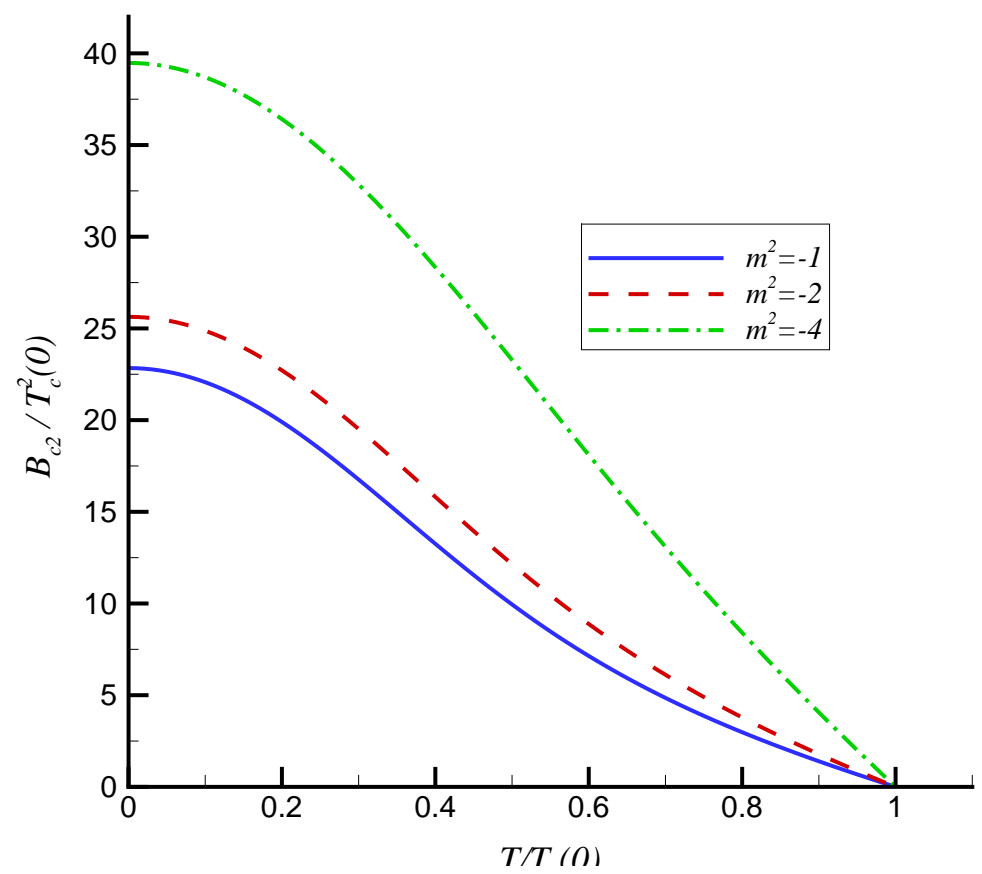

FIG. 2: The behavior of $B_{\mathrm{c} 2} / T_{c}^{2}(0)$ in terms of $T / T_{c}(0)$ for different values of $m^{2}$ and $z_{m}=0.5$, with $d=5$ and $q=5 / 4$. 


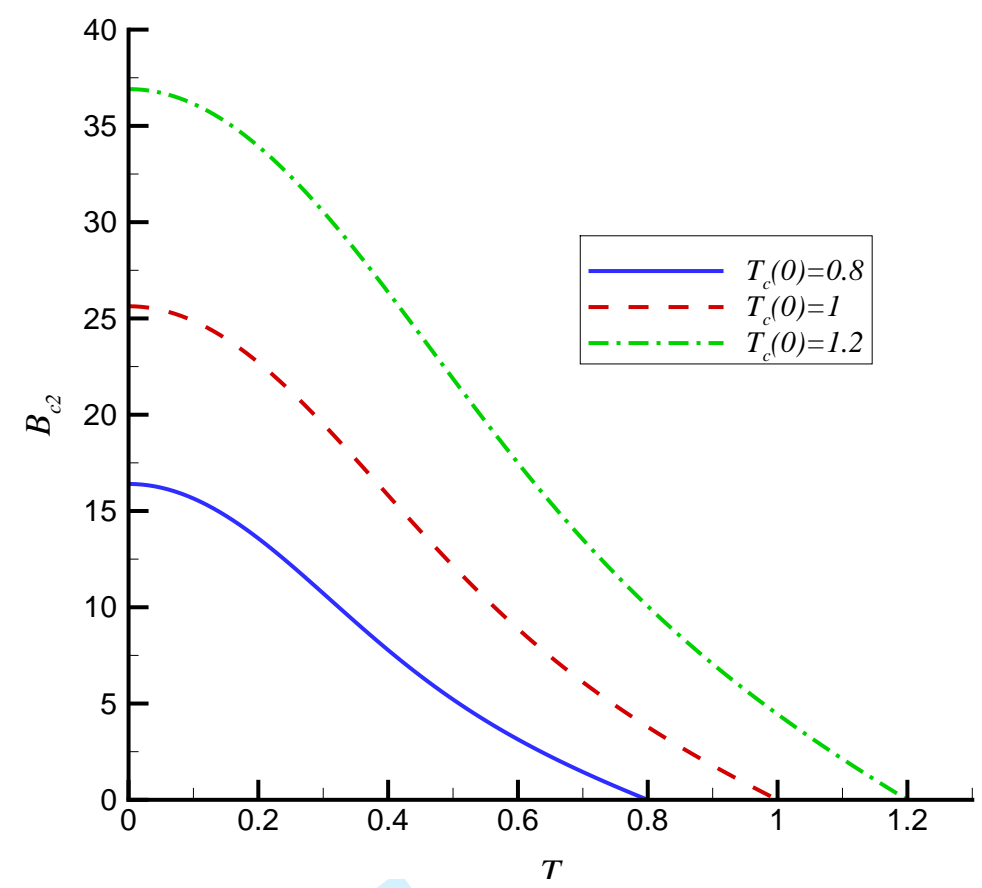

FIG. 3: The upper critical field $B_{c 2}$ as a function of temperature $T$ for different values of $T_{c}(0)$, with $z_{m}=0.5, d=5, q=5 / 4$, and $m^{2}=-2$.

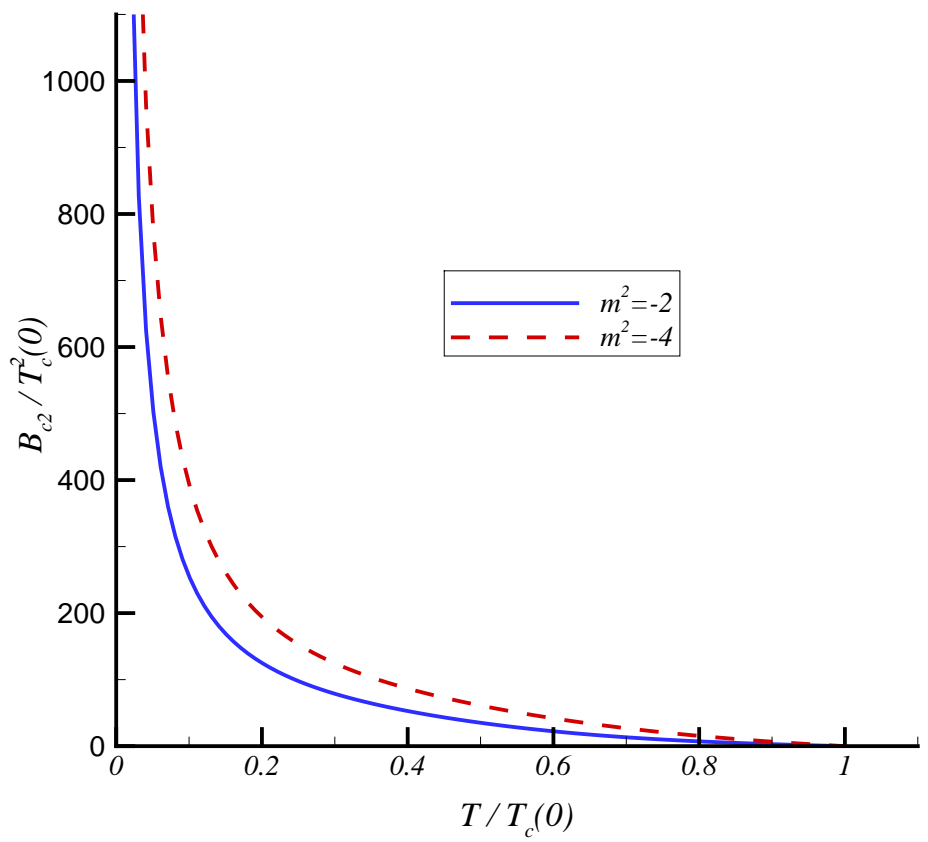

FIG. 4: The behavior of $B_{\mathrm{c} 2} / T_{c}^{2}(0)$ in terms of $T / T_{c}(0)$ for different values of $m^{2}$ and $z_{m}=0.5$, with $d=5$ and $q=1$. 


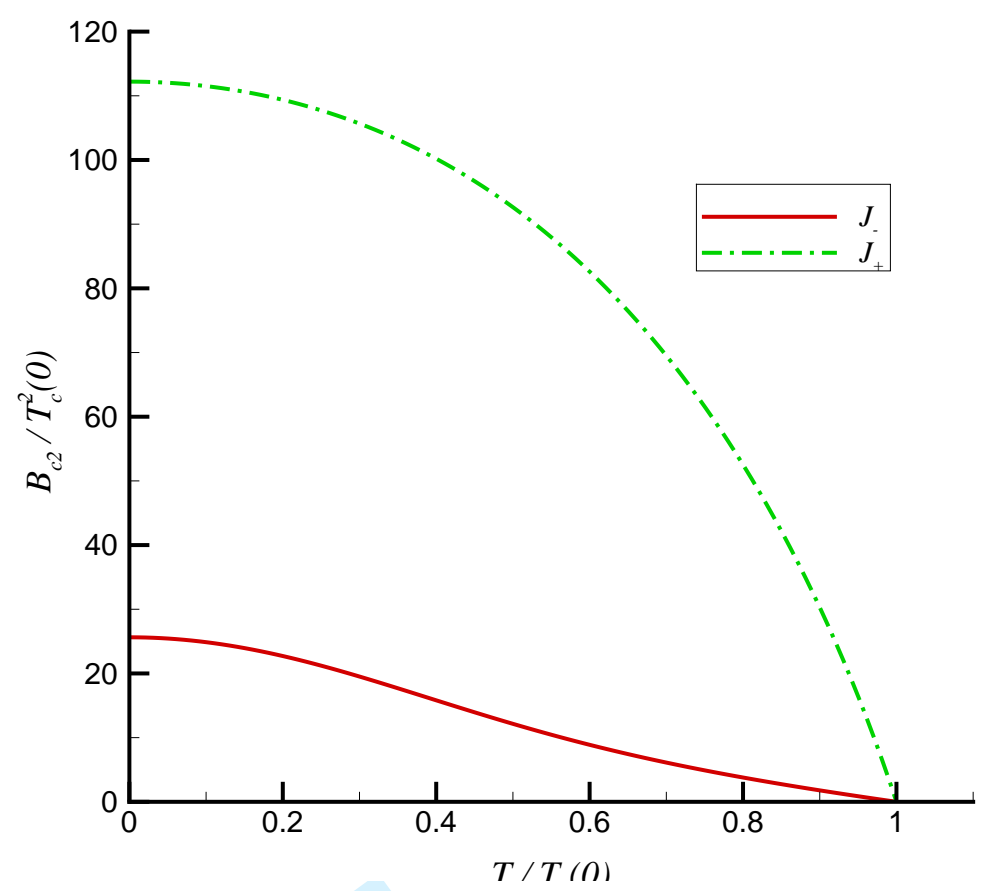

FIG. 5: The behavior of $B_{c 2} / T_{c}^{2}(0)$ in terms of $T / T_{c}(0)$ for different non-zero boundary conditions $J_{-}$and $J_{+}$, with $z_{m}=0.5$, $d=5, q=5 / 4$, and $m^{2}=-2$.

It is important to note that in this case the functional dependence of $B_{\mathrm{c} 2}(T)$ is the same as that of four-dimensional Maxwell case. This is an expected result since for conforamally invariant Maxwell field, the magnetic field in all dimensions has the same behavior as in four dimensions. For $d=5$, expression (57) becomes

$$
\frac{B_{\mathrm{c} 2}(T)}{T_{c}^{2}(0)}=\pi^{2}\left\{\sqrt{\tilde{\omega}^{2}+\left(n^{2}-p\right)\left(\frac{T}{T_{c}(0)}\right)^{4}}-n\left(\frac{T}{T_{c}(0)}\right)^{2}\right\} .
$$

On the other hand if we consider the Maxwell case $(q=1)$ in five dimensions $(d=5)$, then Eq. (56) becomes

$$
\frac{B_{\mathrm{c} 2}(T)}{T_{c}^{2}(0)}=\pi^{2}\left(\frac{T}{T_{c}(0)}\right)^{-1}\left\{\sqrt{\tilde{\omega}^{2}+\left(n^{2}-p\right)\left(\frac{T}{T_{c}(0)}\right)^{6}}-n\left(\frac{T}{T_{c}(0)}\right)^{3}\right\} .
$$

The main difference between expressions of critical magnetic field given in Eq. (58) for the conformally invariant Maxwell field $(d=5, q=5 / 4)$ and the usual Maxwell field $(d=5, q=1)$ given by Eq. (59), is the appearance of the $\left[T / T_{c}(0)\right]^{-1}$ term in the latter case. Clearly this term diverges as $\left[T / T_{c}(0)\right] \rightarrow 0$. The behavior of $B_{\mathrm{c} 2}$ in terms of $T / T_{c}(0)$ for $q=1$ and $d=5$ is shown in Fig. 4. From this figure, it is obvious that the critical magnetic field diverges as $T \rightarrow 0$. This behavior is unacceptable and as we mentioned only the conformally invariant Lagrangian with $q=d / 4$ leads to the desired form of critical magnetic field consistent with the phenomenology of superconductors. Finally, Fig. 5 shows the phase diagrams obtained by imposing the different boundary conditions of non-zero $J_{-}$, as expressed by Eq. (56), and non-zero $J_{+}$, also considered in Ref. [29].

\section{CONCLUSIONS}

In this paper, the properties of $(d-1)$-dimensional $s$-wave holographic superconductor in the presence of conformally invariant power-Maxwell correction to the usual Einstein-Maxwell action, were investigated. It must be noted that the power-Maxwell Lagrangian in $d$ dimensions is invariant under conformal transformation, i.e. $g_{\mu \nu} \rightarrow \Omega^{2} g_{\mu \nu}$ and $A_{\mu} \rightarrow A_{\mu}$, provided we take the power parameter as $q=d / 4$. 
In the absence of external magnetic field, we have found the critical temperature $T_{c}$ to vary as $\rho^{1 /(d-2)}$ for all values of the power parameter $q$, with a proportionality constant $\kappa$ that increases with $q$ or the dimensionality $d$, as expected. The variation of order parameter with the temperature is found to be $<\mathcal{O}>\propto \sqrt{1-\frac{T}{T_{c}}}$ with a critical exponent $\beta=1 / 2$, which is characteristic of systems with mean field behavior, the prime examples of which are the superconductors.

Furthermore, an analytic investigation of the effects of an external magnetic field was made by employing the matching technique. We considered the probe limit in which the scalar and gauge fields do not affect the background metric. The phase behavior of the $(d-1)$-dimensional holographic superconductor was obtained by computing the upper critical magnetic field in the presence of conformally invariant power-Maxwell electrodynamics. It was found that consistent with the phenomenology and Ginzburg-Landau theory of superconductivity, the upper critical field $B_{\mathrm{c} 2}(T)$ vanishes linearly as $T \rightarrow T_{c}(0)$. Also, it became evident that $B_{\mathrm{c} 2}(0)$ increases with $T_{c}(0)$, which is again consistent with the phenomenology of superconductors. The case of physical interest in $d=5$ spacetime dimensions, corresponding to $(3+1)$-dimensional holographic superconductor, was considered in detail with both the conformally invariant $q=5 / 4$ and the usual power-Maxwell parameter $q=1$, and plotted in the figures. Interestingly enough, we observed that in the presence of power-Maxwell electrodynamics, the critical magnetic field $B_{\mathrm{c} 2}$ has reasonable behavior consistent with the phenomenology of superconductors provided we consider the conformally invariant case, i.e. $q=d / 4$. This may be due to the fact that for the conformally invariant power-Maxwell theory, the electromagnetic fields in higher dimensions have the same behavior as in the four-dimensional gravity dual of the well established (2+1)dimensional holographic superconductor [31]. In particular, we found that for $q=1$ and $d=5$, the critical magnetic field $B_{\mathrm{c} 2}$ diverges as $T \rightarrow 0$. This behavior is physically unacceptable and comes from the fact that in the case $q=1$ and $d=5$, the power-Maxwell Lagrangian is not conformally invariant. This point, however, deserves further investigation.

\section{Acknowledgments}

We thank the Research Council of Shiraz University. This work has been supported financially by Research Institute for Astronomy and Astrophysics of Maragha (RIAAM), Iran.

[1] J. M. Maldacena, Adv. Theor. Math. Phys. 2, 231 (1998), [arXiv:hep-th/9711200].

[2] S. A. Hartnoll, Class. Quant. Grav. 26.22 224002(2009), [arXiv:hep-th/0903.3246];

C. P. Herzog, J. Phys. A 42, 343001 (2009), [arXiv:hep-th/0904.1975].

[3] S. A. Hartnoll, C. P. Herzog, and G. T. Horowitz, Phys. Rev. Lett. 101, 031601 (2008), [arXiv:hep-th/0803.3295].

[4] G. Siopsis and J. Therrien, JHEP 05, 013 (2010), [arXiv:hep-th/1003.4275].

[5] R. Gregory, S. Kanno, and J. Soda, JHEP 0910, 010 (2009), [arXiv:hep-th/0907.3203].

[6] S. A. Hartnoll, C. P. Herzog and G. T. Horowitz, JHEP 12, 015 (2008), [arxiv:hep-th/0810.1563].

[7] H. B. Zeng, X. Gao, Y. Jiang, and H. S. Zong, JHEP 1105, 002 (2011), [arXiv:hep-th/1012.5564].

[8] H. F. Li, R. G. Cai, and H. Q. Zhang, JHEP 04028 (2011), [arXiv:1103.2833].

[9] R. G. Cai, H. F. Li, and H. Q. Zhang, Phys. Rev. D 83, 126007 (2011), [arXiv:hep-th/1103.5568].

[10] Q. Y. Pan, B. Wang, E. Papantonopoulos, J. Oliveira, and A. Pavan, Phys. Rev. D 81,106007 (2010), [arXiv:hepth/0912.2475].

[11] R. G. Cai, H. Zhang, Phys. Rev. D 81, 066003 (2010), [arXiv:hep-th/0911.4867].

[12] G. T. Horowitz and M. M. Roberts, Phys. Rev. D 78, 126008 (2008), [arXiv:hep-th/0810.1077].

[13] G. T. Horowitz and M. M. Roberts, JHEP 0911015 (2009), [arXiv:hep-th/0908.3677].

[14] Q. Pan, J. Jing, and B. Wang, JHEP 1111, 088 (2011), [arXiv:hep-th/1105.6153].

[15] R. G. Cai, Z. Y. Nie, H. Q. Zhang, Phys. Rev. D 82, 066007 (2010), [arXiv:hep-th/1007.3321].

[16] Q. Pan and B. Wang, Phys. Lett. B 693, 159 (2010), [arXiv:hep-th/1005.4743].

[17] T. Albash and C. V. Johnson, JHEP 09, 121 (2008), [arXiv:hep-th/0804.3466].

[18] M. Tinkham, Introduction to Superconductivity, 2nd edition, Dover Publications, New York, 1996.

[19] E. Nakano and Wen-Yu Wen, Phys. Rev. D 78, 046004 (2008), [arXiv:0804.3180].

[20] A. Sheykhi, F. Shaker, Phys. Lett. B 754, 281 (2016), [arXiv:1601.05817];

A. Sheykhi, F. Shaker, Int. J. Mod. Phys. D Vol. 26, No. 5, 1750050 (2017), [arXiv:arXiv:1606.04364];

A. Sheykhi, F. Shaker, Cana. J. Phys. 94 (12), 1372 (2016), [arXiv:1601.05817].

[21] Z. Zhao, Q. Pan, S. Chen and J. Jing, Nucl. Phys. B 871, 98 (2013), [arXiv:1212.6693].

[22] S. Gangopadhyay, D. Roychowdhury, JHEP 05, 002 (2012), [arXiv:1201.6520].

[23] T. Albash and C. V. Johnson, [arXiv:0906.0519].

[24] D. Roychowdhury, Phys. Rev. D, 86, 106009 (2012), [arXiv:1211.0904]. 
[25] S. Gangopadhyay, Mod. Phys. Lett. A 29, 1450088 (2014), [arXiv:1311.4416].

[26] S. L. Cui, Z. Xue, Phys. Rev. D 88, 107501 (2013), [arXiv:1306.2013].

[27] S. Dey, A. Lala, Annals of Physics 354, 165 (2015), [arXiv:1306.5137].

[28] A. Sheykhi, F. Shamsi, Int. J. Theor. Phys. DOI 10.1007/s10773-016-3234-1, [arXiv:1603.02678].

[29] D. Roychowdhury, Phys. Lett. B 718, 1089 (2013), [arXiv:hep-th/1211.1612].

[30] M. Hassaine and C. Martinez, Phys. Rev. D 75, 027502 (2007), [arXiv:hep-th/0701058];

H. A. Gonzalez, M. Hassaine and C. Martinez, Phys. Rev. D 80, 104008 (2009), [arXiv:hep-th/0909.1365].

[31] A. Sheykhi, Phys. Rev. D 86, 024013 (2012) [arXiv:1209.2960].

[32] A. Sheykhi, H. R. Salahi and A. Montakhab, JHEP 04, 058 (2016) [arXiv:1603.00075];

H. R. Salahi, A. Sheykhi and A. Montakhab, Eur. Phys. J. C 76, 575 (2016), [arXiv:1608.05025].

[33] J. Jing, Q. Pan, S. Chen, JHEP 11, 045 (2011), [ arXiv:1106.5181].

[34] J. Jing, L. Jiang and Q. Pan, Class. Quantum Grav. 33, 025001 (2016).

[35] M. Ammon, J. Erdmenger, M. Kaminski, P. Kerner, JHEP 0910, 067 (2009), [arXiv:0903.1864];

M. Ammon, J. Erdmenger, M. Kaminski, P. Kerner, Phys. Lett. B 680, 516 (2009), [arXiv:0810.2316];

D. Momeni, M. Raza, R. Myrzakulov, J. of Gravity 2013, 782512 (2013), [arXiv:1305.3541];

D. Momeni, Eiji Nakano, M. R. Setare, Wen-Yu Wen, Int. J. Mod. Phys. A 28, 1350024 (2013),[arXiv:1108.4340].

[36] L. Barclay, R. Gregory, S. Kanno, and P. Sutcliffe, JHEP 1012, 029 (2010) [arXiv:hep-th/1009.1991].

[37] S. Kanno, Class. Quant. Grav. 28,127001, (2011), [arXiv:hep-th/1103.5022].

[38] Xiao-Mei Kuang, Wei-Jia Li, and Yi Ling, JHEP 12, 069 (2010), [arXiv:hep-th/1008.4066]. 